phys. stat. sol. (b) 221, 473 (2000)

Subject classification: $63.20 . \mathrm{Kr} ; 78.47 .+\mathrm{p} ; \mathrm{S} 7.12$

\title{
Dephasing Due to Carrier-Carrier Scattering in 2D
}

\author{
W. A. HÜGEL, M. F. HeINRICH, and M. WEGENER \\ Institut für Angewandte Physik, Universität Karlsruhe (TH), Kaiserstraße 12, \\ D-76128 Karlsruhe, Germany
}

(Received April 10, 2000)

\begin{abstract}
We show that there is no significant difference in the dephasing due to carrier-carrier scattering between bulk GaAs and GaAs quantum wells. This is in contrast to previous experimental work with worse dynamic range.
\end{abstract}

Carrier scattering and screening of the Coulomb potential in two dimensions (2D) are often believed to be quite different from those in three dimensions (3D). Our intuition tells us that carriers in $2 \mathrm{D}$ cannot move in one spatial direction therefore one is tempted to argue that screening of the Coulomb potential will be less effective in 2D. This in turn should strongly influence the carrier-carrier scattering rates via the carrier scattering cross section. Early experiments by Shank and co-workers [1] on quasi-2D GaAs quantum well structures indeed indicated that the decay time $\tau$ of the coherent four-wave mixing (FWM) signal has a scaling with the optically excited carrier density $n_{\mathrm{eh}}$ according to $\tau^{-1} \propto n_{\mathrm{eh}}^{0.55 \pm 0.04}$ which is different from $\tau^{-1} \propto n_{\mathrm{eh}}^{0.3}$, which was found for bulk GaAs by the same group [2]. The results for bulk GaAs were confirmed by us [3] and others [5]. In this article, we present data on GaAs quantum wells under conditions which are comparable to bulk. We find no significant difference between 3D and 2D.

The experiment on quantum wells is very closely similar to our recent experiments on bulk GaAs [3]. The laser spectrum of the nearly transform-limited $11 \mathrm{fs}$ optical pulses is centered slightly above the 2D band edge of the $11.6 \mathrm{~nm}$ wide GaAs quantum well sample [4]. In order to compare our 2D with our 3D experiments (E) [3] and theory (T) [4] we plot the decay time $\tau$ of the time-integrated FWM signal as a function of the optically excited carrier density in Fig. 1. The sheet densities for GaAs quantum wells and the volume densities for bulk GaAs, can simply be related by the QW thickness $L_{z}$ via the relation $n_{\mathrm{eh}}^{(2 \mathrm{D})}=L_{z} n_{\mathrm{eh}}^{(3 \mathrm{D})}$, with $L_{z}=11.6 \mathrm{~nm}$. Obviously, all data points - experiment/theory and 3D/2D - fall together. Note, that we use a logarithmic scale for $n_{\mathrm{eh}}$. From this plot we conclude that there is no significant difference in the dephasing due to carrier-carrier scattering between $3 \mathrm{D}$ and $2 \mathrm{D}$. This is in sharp contrast to previous work [1]. Thus, the following questions arise: (i) How does the quality of our experimental data compare to that of previous work? (ii) Are the excitation conditions (3D/2D) comparable? (iii) What is the role of the second subband? (iv) Is there a dependence on quantum well width?

We now address these issues (i) to (iv). (i) How does the quality of our experimental data compare to that of previous work? The dynamic range in the carrier densities investigated spans two orders of magnitude for both, our experiments on bulk GaAs and our experiments on GaAs quantum wells. In Ref. [1] data are presented over only 


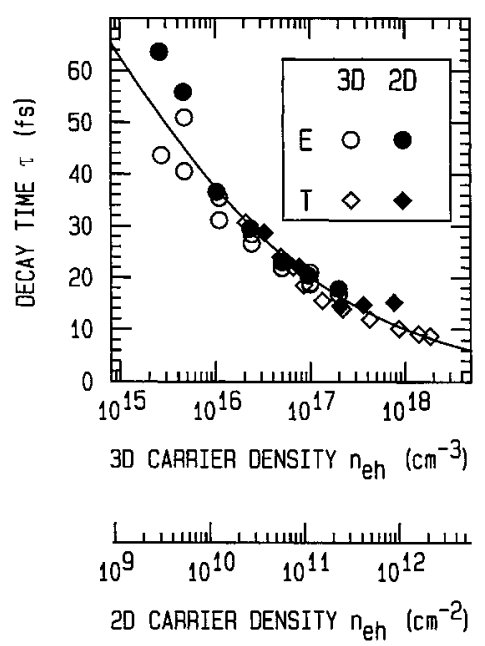

Fig. 1. Decay time $\tau$ versus carrier density for $3 \mathrm{D} / 2 \mathrm{D}$ and experiment/theory $(E / T)$. The four different symbols are explained in the inset. The experiment corresponds to quantum well sample A with well width $L_{z}=11.6 \mathrm{~nm}$. We have used the relation between $2 \mathrm{D}$ and $3 \mathrm{D}$ carrier densities $n_{\mathrm{eh}}^{(2 \mathrm{D})}=L_{z} n_{\mathrm{eh}}^{(3 \mathrm{D})}$. Beside some scatter of the data, all data points fall on one curve, which is drawn as a guide to the eye. This shows that there is no significant difference in the dephasing due to carrier-carrier scattering between $2 \mathrm{D}$ and 3D

one order of magnitude in carrier density, also on GaAs quantum wells. This improvement has simply become possible because we use a laser system with $100 \mathrm{MHz}$ repetition rate while they used a $10 \mathrm{kHz}$ system. Because of the same reason, our FWMsignal versus time delay (from which $\tau$ is obtained) always spans at least two orders of magnitude in signal strength. This was not the case in the work of Shank's group [1]. (ii) Are the excitation conditions (3D/2D) comparable? The excitation conditions in our $3 \mathrm{D} / 2 \mathrm{D}$ experiments are very closely similar. In fact, we have only changed the sample in one setup. Beside the $2 \mathrm{D}$ confinement energy, which is small as compared to the laser bandwidth, the excitation conditions are the same. This is in contrast to Refs. [1,2]. In the bulk case they used excitation at $2 \mathrm{eV}$ photon energy, that is more than $0.5 \mathrm{eV}$ above the GaAs band edge, while their experiments on GaAs quantum well were resonant with the band edge. (iii) What is the role of the second subband? From the FWM spectra [4] it becomes obvious that the second quantum well subband contributes much less to the total signal than the first subband. Thus, it is justified to interpret these results in terms of a quasi-2D system. In Ref. [1] a closely similar quantum well sample was used. (iv) Is there a dependence on quantum well width? We have investigated three different samples: All samples are high quality $\mathrm{Al}_{x} \mathrm{Ga}_{1-x} \mathrm{As} / \mathrm{GaAs}$ heterostructures grown by molecular-beam epitaxy on GaAs substrates. Sample A contains 20 periods of $11.6 \mathrm{~nm}$ wide GaAs quantum wells and $15.0 \mathrm{~nm}$ thick $\mathrm{Al}_{x} \mathrm{Ga}_{1-x} \mathrm{As}$ $(x=0.3)$ barriers. Its substrate was etched off. Then, the sample was glued to a sapphire substrate on one side and was antireflection coated with a single quarter wave layer corresponding to the wavelength of the band edge of the quantum wells on the other side. The exciting laser pulses first pass this sapphire substrate (the dispersion of which is carefully precompensated) before they hit the quantum wells, such that the FWM signal does not experience any additional group velocity dispersion. At $T=77 \mathrm{~K}$, sample A exhibits a heavy-hole damping of $1.5 \mathrm{meV}$ as measured in the linear absorption spectrum. All experiments reported here have been performed at room temperature where the linewidth is larger than this value and we can safely rule out any influence from undesired inhomogeneous broadening for the results discussed here. This makes our results different from those in Ref. [6] which are governed by a photon echo 
response due to inhomogeneously broadened excitonic transitions originating from intentional fluctuations among the various quantum wells. Sample B contains 60 periods of $7.9 \mathrm{~nm} \mathrm{GaAs}$ wells and $11.1 \mathrm{~nm} \mathrm{Al}_{x} \mathrm{Ga}_{1-x}$ As barriers with an aluminum content of $x=0.34$. Sample C contains 60 periods of $5.0 \mathrm{~nm} \mathrm{GaAs}$ wells and $15.0 \mathrm{~nm} \mathrm{Al}_{x} \mathrm{Ga}_{1-x} \mathrm{As}$ barriers with an aluminum content of $x=0.3$. The heavy-hole exciton resonance at room temperature is observed in the FWM spectra at a photon energy $\hbar \omega=1.445 \mathrm{eV}$ for sample $\mathrm{A}$, at $\hbar \omega=1.459 \mathrm{eV}$ for sample $\mathrm{B}$, and at $\hbar \omega=1.491 \mathrm{eV}$ for sample C. Figure 2 summarizes our results on samples A, B, and C. In Fig. 2, (a) corresponds to our sample A, (b) to sample B, and (c) to sample C. The exciting laser spectrum is the same for all three cases, while the band edge of the quantum wells shifts as it has just been discussed. Part (d) of Fig. 2 finally reproduces the data published in Ref. [1]. The sample of that study had $9.6 \mathrm{~nm}$ GaAs wells and $9.8 \mathrm{~nm} \mathrm{Al}_{x} \mathrm{Ga}_{1-x}$ As barriers with $x=0.3$. Unfortunately, the authors do not quote the number of quantum well periods of their sample. From their measured optical density of 0.3 in the band-to-band continuum [1] one can estimate that the number of periods has to be around 30. Their excitation conditions are roughly similar, however, their square-shaped spectra tend to excite somewhat higher in the band-to-band continuum [1]. This might change the relative contributions of excitons and 'free carriers'. Unfortunately, they measured no

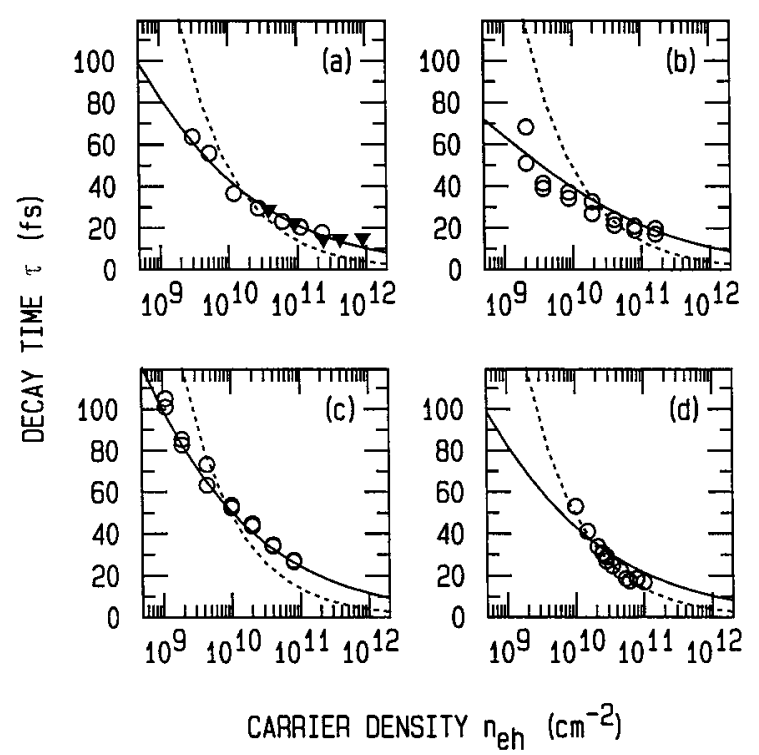

Fig. 2. Experiment/theory: Decay time $\tau$ of the time-integrated FWM signal versus the logarithm of the optically excited sheet carrier density per quantum well $n_{\mathrm{eh}}, T=300 \mathrm{~K}$. Experiment (open circles): (a) sample A (11.6 nm well width), (b) sample B (7.9 nm well width), and (c) sample C (5.0 nm well width); (d) is the complete experimental data of Ref.[1]. The dashed curve in (a) to (d) is the fit given in Ref. [1] according to the scaling $\tau^{-1}=c n_{\mathrm{eh}}^{0.55 \pm 0.04}$. The solid lines are our fits according to $\tau^{-1}=\tau_{0}^{-1}+c n_{\mathrm{eh}}^{1 / 3}$. Here we have used $\tau_{0}=400 \mathrm{fs}$ and $c=9.6 \times 10^{-6} \mathrm{fs}^{-1} \mathrm{~cm}^{2 / 3}$ for sample A, $\tau_{0}=140 \mathrm{fs}$ and $c=8.5 \times 10^{-6} \mathrm{fs}^{-1} \mathrm{~cm}^{2 / 3}$ for sample B, and $\tau_{0}=640 \mathrm{fs}$ and $c=8.5 \times 10^{-6} \mathrm{fs}^{-1} \mathrm{~cm}^{2 / 3}$ for sample C. For comparison, the solid line in (d) is identical to the solid line in (a). Theory (full triangles in (a)): decay times $\tau$ obtained from fitting exponential decays to the numerically computed FWM signal. Note the good quantitative agreement with experiment over about one order of magnitude in carrier density 
FWM spectra which could be compared directly with our FWM spectra [4] in order to answer this question. Figure 2 also shows a number of fits for comparison. Part (d) shows the fit given in Ref. [1] to their data according to $\tau^{-1}=c n_{\mathrm{eh}}^{0.55}$ (dashed lines in (a) to (d)). This fit is not compatible with ours according to $\tau^{-1}=\tau_{0}^{-1}+c n_{\mathrm{eh}}^{1 / 3}$ (solid lines in (a) to (c)) - in agreement with our above discussion. As the actual values for $\tau_{0}$ are rather large $(400 \mathrm{fs}, 140 \mathrm{fs}$, and $640 \mathrm{fs}$ for samples $\mathrm{A}, \mathrm{B}$, and $\mathrm{C}$, respectively), these curves are not very much different from the scaling $\tau^{-1} \propto n_{\mathrm{eh}}^{1 / 3}$. In other words: the fit is not very sensitive to $\tau_{0}$, thus we obtain a considerable scatter in the values of $\tau_{0}$ for the different samples (caption of Fig. 2).

To avoid confusion: we do not claim that from the fits one could distinguish whether the exponent is $1 / 2$ or $1 / 3$. In fact, one cannot. We do state, however, that their conclusion [1] is inconsistent with ours. Our conclusion is that there is no significant difference in the dephasing due to carrier-carrier scattering between 3D and 2D. Figure 2 strongly supports this claim.

In summary, we have shown that there is no significant difference in the dephasing due to carrier-carrier scattering between 3D (bulk GaAs) and 2D (GaAs quantum wells) at room temperature. This conclusion is in contrast to the one of Ref. [1]. We have argued that our experimental data are more complete. Our conclusion does, however, agree with that drawn from previous pump/probe experiments using $120 \mathrm{fs}$ pulses, which directly compared 3D and 2D InGaAs samples [7]. From these experiments no difference was found in the carrier thermalization and carrier cooling due to carriercarrier scattering between $3 \mathrm{D}$ and $2 \mathrm{D}$. This complements our experiments which are specifically sensitive to dephasing.

Acknowledgement This work has been supported by the DFG-SPP "Quantenkohärenz in Halbleitern" and by the Krupp-Stiftung. The reaserch of M.W. is supported by the Leibniz-Preis of the DFG. We thank G. Weimann for the high quality GaAs QW sample. We acknowledge many stimulating discussions with H. Haug and L. Banyai.

\section{References}

[1] J. Y. Bigot, M.T. Portella, R. W. Schoenlein, J.E. Cunningham, and C. V. Shank, Phys. Rev. Lett. 67, 636 (1991).

[2] P. C. Becker, H. L. Fragito, C. H. Brito Cruz, R. L. Fork, J.E. Cunningham, J.E. Henry, and C. V. SHANK, Phys. Rev. Lett. 61, 1647 (1988).

[3] W. A. Hügel, M. F. Heinrich, M. Wegener, Q. T. Vu, L. Bányai, and H. Haug, Phys. Rev. Lett. 83, 3313 (1999).

[4] B. Mieck, H, Haug, W. A. Hügel, M. F. Heinrich, and M. Wegener, Phys. Rev. B, submitted (2000).

[5] S. Arlt, U. Siegner, J. Kunde, F. Morier-Genoud, and U. Keller, Phys. Rev. B 59, 14860 (1999).

[6] J. P. Likforman, M. Joffre, and V. Thierry-Mieg, Opt. Lett. 22, 1104 (1997).

[7] S. Bolton, G. Sucha, and D. S. Chemla, Phys. Rev. B 58, 16326 (1998).

[8] D. W. SNOKE, private communications (1999). 